\title{
Análise exploratória de dados de imagens digitais noturnas
}

\author{
Exploratory Analysis of Nocturnal Digital Images \\ C. R. Steffens ${ }^{1 *}$; P. L. J. Drews-Jr' ${ }^{1}$, S. S. C. Botelho ${ }^{1}$, V. L. D. Mattos ${ }^{2}$ \\ ${ }^{I}$ Centro de Ciências Computacionais, Universidade Federal do Rio Grande, 96203-900, Rio Grande-RS, Brasil \\ ${ }^{2}$ Instituto de Matemática, Estatística e Física, Universidade Federal do Rio Grande, 96203-900, Rio Grande-RS, Brasil \\ *cristianosteffens@furg.br
}

\begin{abstract}
Em aquisição de imagens, o triângulo da exposição é fator determinante para a qualidade percebida e captura fidedigna dos elementos que compõe a cena. Condições de aquisição não controladas ou ambientes que não permitem a utilização de iluminação artificial adequada resultam em imagens que demandam pósprocessamento e correção. Neste trabalho faz-se uma análise baseada em estatística inferencial sobre os impactos da subexposição e superexposição na distribuição da luminância mensurada. Dois conjuntos pareados de imagens noturnas são considerados, modificando-se o tempo de exposição. Os resultados obtidos indicam que a distribuição dos valores de píxel é afetada de maneira significativa, indicando a possibilidade do uso de estatísticas clássicas para compreender e mitigar os efeitos indesejáveis da saturação e subexposição. Com base nos resultados, considera-se viável a aplicação de estatísticas clássicas como função objetivo em redes neurais artificiais profundas, oferecendo vantagens, em termos de custo computacional, sobre técnicas baseadas na perda perceptual ou no treinamento de redes adversárias.

Palavras-chave: Processamento de Imagens, Estatísticas de Imagem, Aprimoramento de Imagens
\end{abstract}

In image acquisition, the exposure triangle is a determining factor for the perceived quality and reliable capture of the elements that make up the scene. Uncontrolled acquisition conditions, as well as environments that do not allow the use of adequate artificial lighting, result in images that require post-processing and correction. In this work, an analysis based on inferential statistics on the impacts of underexposure and overexposure on the measured luminance distribution is made. Two paired sets of night images are considered by modifying the exposure time. The results indicate that the distribution of pixel values is significantly affected, indicating the possibility of using classical statistics to understand and mitigate the undesirable effects of saturation and underexposure. Based on the results, it is considered feasible to apply classical statistics as an objective function in deep artificial neural networks, offering advantages, in terms of computational cost, of techniques based on perceptual loss or training of adversary networks.

Keywords: Image Processing, Image Statistics, Image Enhancement

\section{INTRODUÇÃO}

No decorrer das últimas décadas, sistemas baseados em tecnologias de percepção computacional têm ganhado relevância em diversas aplicações. Atualmente é comum que indústrias e até mesmo usuários domésticos façam uso de sistemas de reconhecimento de voz, sistemas de reconhecimento de caracteres, ou mesmo sistemas de busca baseada em imagem. Algoritmos de reconhecimento, classificação e segmentação semântica de imagens, de maneira pervasiva, fazem parte do cotidiano das pessoas.

Apesar da relevância e ampla utilização de sistemas baseados em vídeo, faz-se necessário destacar que sensores de imagem apresentam sérias restrições quanto à sua capacidade de capturar as características da cena. Estas restrições ficam mais claras quando se considera, por exemplo, uma cena com iluminação insuficiente, uma cena com objetos de superfície reflexiva, ou até mesmo uma cena com alto contraste. É comum deparar-se com imagens digitais onde alguma parte do conteúdo não está apropriadamente visível.

A física desempenha um papel fundamental para a compreensão deste fenômeno. A luz é o fator mais importante para a aquisição de imagens no espectro da luz visível. Esta afirmação pode ser verificada ao considerar-se o modelo matemático de câmera pinhole [1]. As restrições são dadas pelo triângulo da exposição: ISO (termo genericamente empregado para descrever os padrões de velocidade e sensibilidade introduzidos nas ISO 2240:2003, ISO 6:1993 e ISO 12232:2006), tempo 
de exposição e abertura. O ISO representa um ganho aplicado ao sensor. Ao aumentar-se o ISO aumenta-se a sensibilidade do sensor à luz, refletindo, no entanto, em maior granularidade (ruído) visível na imagem adquirida. Ao aumentar-se a abertura da íris da lente, aumenta-se a quantidade de luz que alcança o sensor. Esta medida tem como efeito adverso a perda de profundidade de campo. Por fim, o tempo de exposição corresponde ao período pelo qual o sensor permanece recebendo luz para compor uma única imagem, refletindo-se em borramento para cenas não estáticas. Em aplicações de vídeo, o tempo de exposição é usualmente limitado em 33.33 milissegundos, que correspondem a taxa de 30 quadros por segundo.

Uma das motivações do presente trabalho, é investigar a viabilidade de uso de estatísticas clássicas como função objetivo para o treinamento de modelos de restauração de imagens baseados em redes neurais convolucionais $(\mathrm{CNN})$. Estes modelos, por padrão, requerem amostras pareadas para treinamento, onde cada amostra é composta por uma entrada e uma saída esperada. Para casos em que não é possível obter dados pareados, Goodfellow et al. (2014) [2] apresenta o conceito de GAN's (Generative Adversarial Network), que combina duas redes neurais no arranjo gerador-discriminador. A rede discriminadora aprende a classificar dados entre duas classes (real, falsa). Já a rede geradora aprende a enganar a rede discriminadora, e gerar um dado classificado como real, de forma que ambas aprendam de maneira conjunta. Ao final do treinamento, apenas a rede geradora é utilizada para aplicação. Apesar de obter excelentes resultados, o treinamento de GAN's tem um custo alto custo computacional, uma vez que duas redes neurais precisam ser treinadas ao mesmo tempo.

Este artigo explora como as características perceptíveis de uma imagem afetam suas propriedades estatísticas. De forma geral, busca-se verificar se é possível identificar estatísticas que permitam diferir uma imagem apropriadamente exposta de uma imagem inadequadamente exposta. Para tanto, faz-se o uso de Análise Inicial de Dados (AID) e Análise Exploratória de Dados (AED) de modo a identificar as características principais da amostra e verificar os pressupostos exigidos para ajuste de modelos e teste de hipóteses.

\section{TRABALHOS RELACIONADOS}

A relação entre as estatísticas de uma imagem e as suas propriedades visuais aparentes vem sendo explorada por diversos autores. Destacam-se aqui trabalhos relacionados relevantes, que fornecem os subsídios teóricos, experimentais e metodológicos para o desenvolvimento do presente trabalho. Entre outros, as propriedades estatísticas de imagem vêm sendo exploradas com a finalidade de aumentar a compreensão sobre a estrutura do sistema visual humano, desenvolver técnicas de representação e codificação eficientes e verificar como as características estão associadas com as propriedades visíveis de superfícies e estruturas.

Simoncelli e Olshausen (2001) [3] utilizam a inferência estatística para identificar as propriedades de imagens naturais (ambiente externo, com pouca intervenção humana) e como estas se refletem no sistema visual primário. Os autores defendem que o desenvolvimento do sistema visual está fortemente atrelado à tarefa que o indivíduo desempenha, a capacidade computacional (quantidade de neurônios, metabolismo, e restrições de conectividade), e ao ambiente em que o organismo habita. Uma vez que existe uma capacidade restrita, é natural assumir que o sistema evolui para se adaptar aos sinais aos quais é exposto com maior frequência. Estas premissas são justificadas a partir da teoria matemática da informação.

Simoncelli e Olshausen (2001) [3] apresentam um estudo de caso para imagens naturais considerando a distribuição não gaussiana de intensidade de luz (luminância), a distribuição dos comprimentos de onda no espectro (cor), a autocorrelação espacial (translação), a correlação no espaço de Fourier, e a dependência espaço-temporal (sequência de quadros). Os resultados obtidos fornecem subsídio para a compreensão estatística da organização das células que compõe o sistema visual.

Torralba e Oliva (2003) [4] estudam as propriedades estatísticas de imagens naturais pertencentes a diferentes categorias e sua relevância para a tarefa de reconhecimento. Os autores discorrem sobre a viabilidade de acelerar algoritmos de classificação de imagens a partir de uma abordagem top-down utilizando informações contextuais já nas primeiras etapas do fluxo de processamento. Para tanto, os autores utilizam uma base de dados de imagem e verificam que uma região específica da imagem fornece a maior parte da informação sobre uma categoria. Para 
exemplificar, informações relacionadas a condição climática ficam concentradas na parte superior da imagem, ao passo que informações sobre pedestres ficam na parte inferior da imagem. Os autores demonstram como estas propriedades podem ser utilizadas para, de maneira simples, prever a presença de objetos na cena dispensando um processamento mais aprofundado da imagem.

Torralba e Oliva (2003) [4] identificam ainda a relação entre diferentes categorias, a orientação e a distribuição de frequência. Os resultados mostram que a diferenciação entre várias categorias feitas pelo homem reside principalmente na relação entre contornos horizontais e verticais em diferentes escalas, enquanto as assinaturas espectrais de ambientes naturais têm uma variação mais ampla em formas espectrais. Isto se deve em muito, ao fato de objetos fabricados pelo homem utilizarem materiais de superfície homogênea e serem constituídos de formas geométricas bem definidas.

Geisler (2008) [5] apresentam uma variação de Simoncelli e Olshausen (2001) [3] com o intuito de desenvolver modelos estatísticos para percepção. Apresenta-se um framework Bayesiano do 'observador ideal' para inferência estatística de modo a caracterizar estatísticas de cenas naturais e avaliar sua conexão com o desempenho em tarefas específicas. A medição de estatísticas de cenas naturais permitiu identificar fontes de estímulo para diferentes tarefas de percepção, bem como determinar as faixas típicas e a confiabilidade dessas fontes de informação.

Sob uma perspectiva diferente, Motoyoshi et al. (2007) [6] exploram a influência das propriedades estatísticas sobre a qualidade percebida de texturas superficiais. $\mathrm{O}$ trabalho avalia como a iluminação, refletância e geometria superficial podem ser identificados observando apenas assimetria e desvio padrão da imagem. Identificou-se que a assimetria do histograma de luminância e a assimetria das saídas do filtro de sub-bandas estão correlacionadas com o brilho da superfície e inversamente correlacionadas com o albedo da superfície (reflectância difusa). Os autores encontraram evidências de que os humanos usam a assimetria, ou uma medida semelhante de assimetria da distribuição, para fazer julgamentos sobre superfícies.

Assumindo premissas semelhantes, He et al. (2011) [7] apresentam um método para supressão dos efeitos de neblina em fotografias. O modelo apresentado explora a característica esbranquiçada da neblina e seu reflexo sobre a curtose. Através de uma abordagem por blocos (patch), o modelo é capaz de restaurar o contraste e minimizar o impacto da perda de transmissão provocada pelas partículas suspensas. Além da supressão dos efeitos de neblina, abordagens posteriores de Miao et al. (2014) [8] e Long et al. (2010) [9] embasaram-se nos mesmos fundamentos para o desenvolvimento de sistemas de detecção de fumaça em vídeo.

Por fim, medidas estatísticas foram ainda utilizadas para avaliação da qualidade de imagem. Wang et al. (2004) [10] e (1998) [11] utilizam média, variância e covariância entre luminância, contraste e estrutura de duas imagens para compor uma métrica capaz de mensurar a perda causada por algoritmos de compressão, bem como os resultados de algoritmos de processamento de imagens, desde que exista uma imagem de referência.

\section{MATERIAL E MÉTODOS}

A avaliação das propriedades estatísticas é conduzida utilizando-se 2 conjuntos de imagens. $O$ intervalo de representação da luminância utilizado é $[0,1]$, onde 0 indica preto e 1 indica branco. A luminância $Y$ para cada píxel é obtida a partir da imagem RGB por $Y=0.299 \times R+0.587 x$ $G+0.114 \times B$. Reforça-se que $Y$ é uma medida abstrata, uma vez que reflete a quantidade de luz convertida em elétrons no sensor.

- Dataset 1: 2697 pares de imagens obtidas com câmera Sony $\alpha 7 \mathrm{~S}$ II, com pares compostos de uma imagem subexposta e uma imagem apropriadamente exposta de ambientes com pouca luz, de Chen et al. (2018) [12]. Os pares são obtidos variandose o tempo de exposição do sensor. A Figura 1 apresenta uma das cenas deste conjunto de dados.

- Dataset 2: 2397 pares de imagens obtidas com câmera Fujifilm X-T2, com pares compostos de uma imagem sub-exposta e uma imagem apropriadamente exposta de ambientes com pouca luz, de Chen et al. (2018) [12]. Os pares são obtidos variandose o tempo de exposição do sensor. A Figura 2 apresenta uma das cenas que compõe este conjunto de dados. Verifica-se, que com este sensor, as imagens subexpostas resultantes apresentam expressivo ruído aparente. 
$\mathrm{O}$ uso de datasets gerados a partir de equipamentos distintos faz-se relevante para identificar a manutenção de premissas entre os diferentes conjuntos de dados. Destaca-se que cada dataset é composto por cenas distintas, sendo que estas retratam, em sua maior parte, cenas urbanas típicas. Para a obtenção das propriedades estatísticas considera-se cada valor de píxel da imagem como uma amostra.
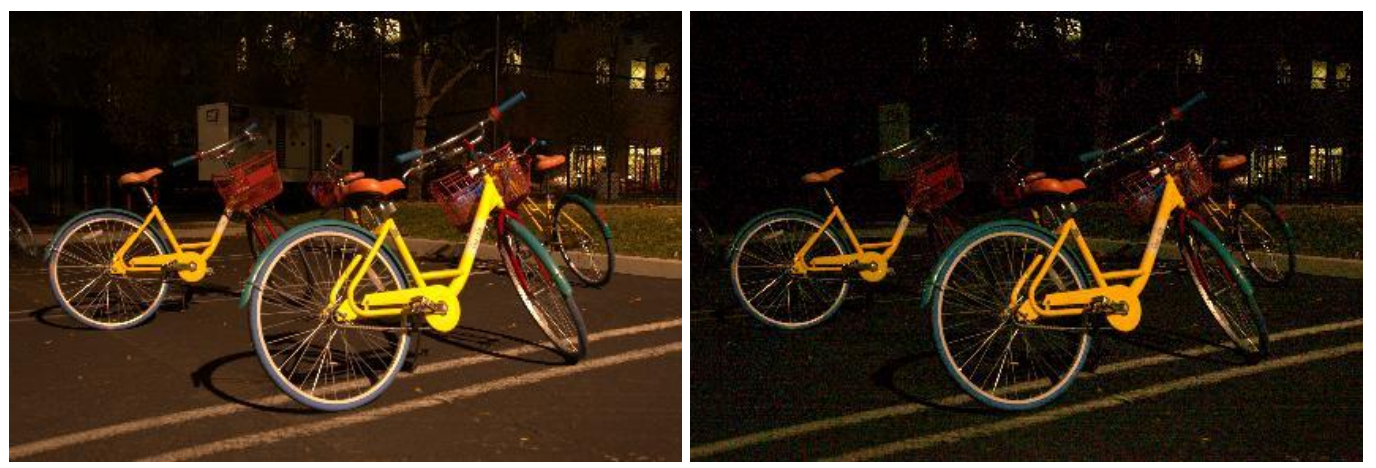

Figura 1: Distribuição dos valores de intensidade de píxel para imagens de cenas do Dataset 1 - Sony para condição adequada e subexposta. Fonte: Chen et al. (2018) [12]
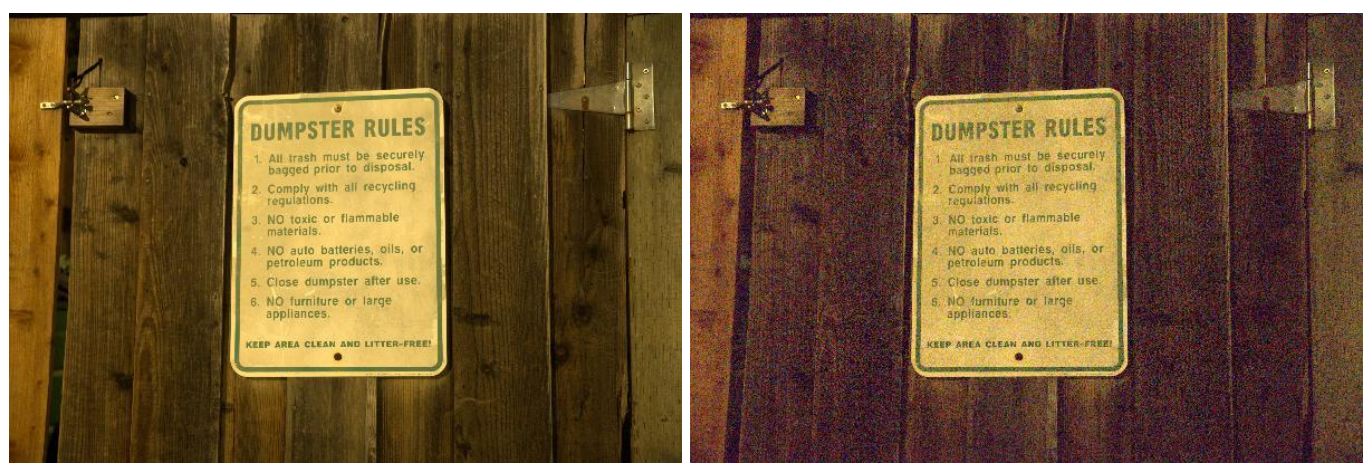

Figura 2: Distribuição dos valores de intensidade de píxel para imagens de cenas do Dataset 2 - Fuji para condição adequada e subexposta. Fonte: Chen et al. (2018) [12]

A maioria das técnicas aplicadas na análise estatística de dados são baseadas em modelos teóricos que pressupõe distribuição normal. A avaliação da normalidade da distribuição dos dados é adequada para a descrição e análise da amostra. Verificando-se que os dados não seguem essa distribuição, o uso de técnicas estatísticas que tem a normalidade como pressuposto, incorre no enviesamento dos parâmetros e da inferência dos testes.

De forma geral, uma primeira avaliação da normalidade de um conjunto de dados pode ser realizada pela observação de seu histograma, identificando assimetrias, descontinuidades de dados e picos multimodais. Como etapa seguinte, com o intuito de verificar a normalidade da série, podese aplicar o teste de Jarque e Bera (1980) [13]. O teste de Jarque e Bera (1980) [13] verifica como o coeficiente de curtose e o coeficiente de assimetria de um conjunto de dados se ajustam aos de uma distribuição normal. Quanto mais próximo de 0 for a estatística JB, maior a probabilidade de a distribuição ser normal. $\mathrm{O}$ teste tem como hipótese nula $\mathrm{H}_{0}$ a normalidade. Desta forma, se o valor-p for menor do que um determinado nível de significância, rejeita-se a normalidade.

$\mathrm{O}$ teste JB de normalidade avalia os estimadores de assimetria e curtose, que representam aspectos relacionados à forma do histograma: desviado para a esquerda/direita (simetria) ou apiculado/achatado (curtose). Combinados com medidas de tendência central e variabilidade podem descrever o conjunto de dados. Jarque e Bera (1980) [13] utiliza como parâmetros os coeficientes de curtose 3 e assimetria 0 . Desvios muito grandes, como, por exemplo, uma curtose acima de 4 e assimetria acima de 1 invalidam a avaliação dos erros-padrão e intervalos de confiança.

Caso seja identificada a não-normalidade dos dados, pode-se fazer o uso de testes não paramétricos, uma vez que em geral não supõem uma distribuição específica para a população. Os modelos paramétricos são sempre preferíveis aos métodos não-paramétricos por serem mais 
poderosos. Nos métodos não-paramétricos há perda de informação pois os dados são trabalhados em forma de ranking, perdendo a magnitude das observações.

Uma vez que cada um dos datasets utilizados é composto por dois subconjuntos distintos (mesma cena retratada de maneira diferente em virtude da modificação dos parâmetros do sistema de aquisição), cabe ainda fazer a avaliação estatística para comparação dos dois conjuntos. São aplicáveis a estes conjuntos de dados técnicas para dados pareados.

Inicialmente, avalia-se a correlação entre os subconjuntos que compõe cada dataset. Uma vez que ambas as imagens representam a mesma cena e pose, é esperado que apresentem associação significativa. O coeficiente de correlação de Pearson mede a dependência linear entre dois conjuntos de dados assumindo distribuição normal. $\mathrm{O}$ valor do coeficiente varia entre - 1 (relação linear inversa perfeita) e +1 (relação positiva perfeita) [14]. O valor-p do teste indica a probabilidade de um sistema não correlacionado produzir datasets que tenham uma correlação pelo menos tão extrema quanto a calculada a partir desses conjuntos de dados. Para fins de interpretação, a correlação é estatisticamente significativa se $p \leq \alpha$.

$\mathrm{O}$ teste de Pearson é, em geral, adequado para dados que estão em um intervalo bem determinado, onde a unidade tem significado por si só (i.e. temperatura em $\mathrm{C}^{o}$, comprimento em metros). Uma vez que esta condição não reflete adequadamente os dados utilizados neste estudo, faz-se o uso também do coeficiente de correlação de postos de Spearman. A correlação de Spearman é uma medida não paramétrica da monotonicidade (as variáveis movem-se na mesma direção relativa) da relação entre dois conjuntos de dados. Ao contrário da correlação de Pearson, a correlação de Spearman não assume que ambos os conjuntos de dados sejam normalmente distribuídos. Correlações de -1 ou +1 implicam em um relacionamento monotônico exato.

Faz-se uso ainda do teste não-paramétrico de Ansari e Bradley (1960) [15] para testar a hipótese nula $\mathrm{H}_{0}$ de que as funções de distribuição da população correspondentes às duas amostras são idênticas. Segundo Sprent e Smeeton (2000) [16] este teste é uma alternativa não paramétrica ao teste $\mathrm{F}$ de duas amostras com variâncias iguais. Ansari e Bradley (1960) [15] não requer a suposição de que os dados vêm de distribuições normais, no entanto exige que as amostras tenham medianas iguais. Sob essa suposição, e se as distribuições das amostras forem contínuas e idênticas, o teste é independente das distribuições.

$\mathrm{O}$ teste $\mathrm{T}$ de Student pareado, mede se a pontuação média difere significativamente entre as amostras. Trata-se de um teste paramétrico que pressupõe normalidade. Se observarmos um valorp maior que o nível de significância, então não podemos rejeitar a hipótese nula $\mathrm{H}_{0}$ de pontuações médias idênticas. Se o valor-p for menor que o nível de significância rejeita-se a hipótese nula de médias iguais. Neste trabalho, aplicou-se o teste bilateral, com $H_{0}: \mu D=0$ e $H_{l}: \mu D \neq 0$.

Uma vez que os dados não apresentem normalidade, é apropriada a utilização de um teste nãoparamétrico como o teste $\mathrm{T}$ de Wilcoxon. $\mathrm{O}$ teste dos postos sinalizados de Wilcoxon testa a hipótese nula de que duas amostras pareadas relacionadas provêm da mesma distribuição. $\mathrm{O}$ teste $\mathrm{T}$ de Wilcoxon tem como hipótese nula $\mathrm{H}_{0}: \mathrm{MD}=0$, onde $\mathrm{M}$ representa a mediana da diferença. Tratando-se de um teste que utiliza o posto da diferença, pode-se utilizar duas abordagens para as diferenças zero: $i$ ) na abordagem de Pratt, mais conservadora, diferenças zeros são consideradas no ranqueamento; ii) na abordagem de Wilcoxon, diferenças zero são descartadas.

Na comparação entre os subconjuntos subexposta--adequada que compõe cada um dos datasets, utilizou-se ainda o teste Levene [17] para variâncias iguais. Este teste é adequado para conjuntos de dados onde a hipótese de normalidade é rejeitada. Neste teste, tem-se a hipótese nula $H_{0}$ : $\sigma^{2}{ }_{l}=\sigma_{2}^{2}=\cdots=\sigma_{k}^{2}$ e hipótese alternativa $H_{l}: \sigma_{i}^{2} \neq \sigma_{j}^{2}$ para pelo menos um par $(i, j)$. Utiliza-se nesta pesquisa a abordagem estendida de Brown e Forsythe (1974) [18], utilizando a mediana como tendência central. A abordagem baseada na mediana é recomendada por oferecer maior robustez para dados não normais.

\section{RESULTADOS E DISCUSSÃO}

Os histogramas e diagramas de caixa apresentados nas Figuras 3 e 4 mostram a distribuição dos valores de pixel nas imagens subexpostas considerando os 2 datasets. Percebe-se uma maior concentração dos valores na região próxima a 0 , indicando que, de maneira geral a imagem é bastante escura. Destaca-se que as imagens retratam cenas noturnas com pouca iluminação. Pelo diagrama de caixas é possível identificar que o Dataset 2: Fuji (Figura 4) apresenta variabilidade 
maior que o Dataset 1: Sony (Figura 3), sendo que em ambos os casos a distância do primeiro quartil para a mediana é menor que a distância da mediana até o terceiro quartil. Esta observação reflete forte assimetria nos valores de intensidade de píxel.
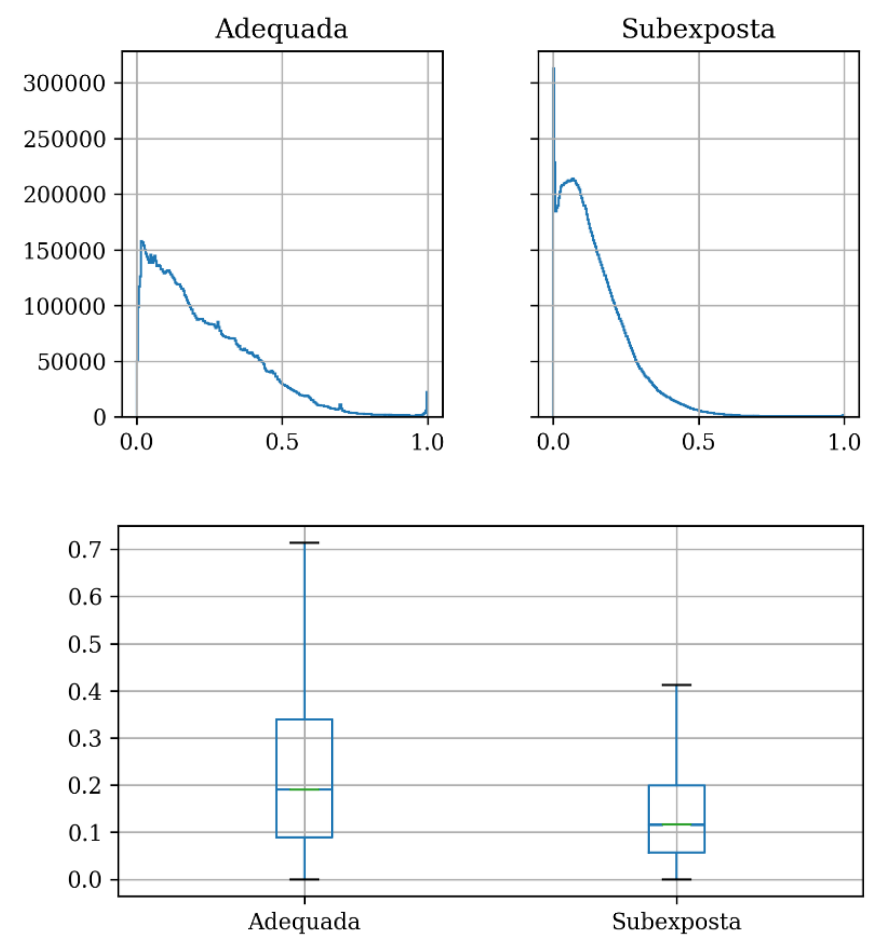

Figura 3: Distribuição dos valores de intensidade de píxel para imagens de cenas do Dataset 1: Sony para condição adequada e subexposta.
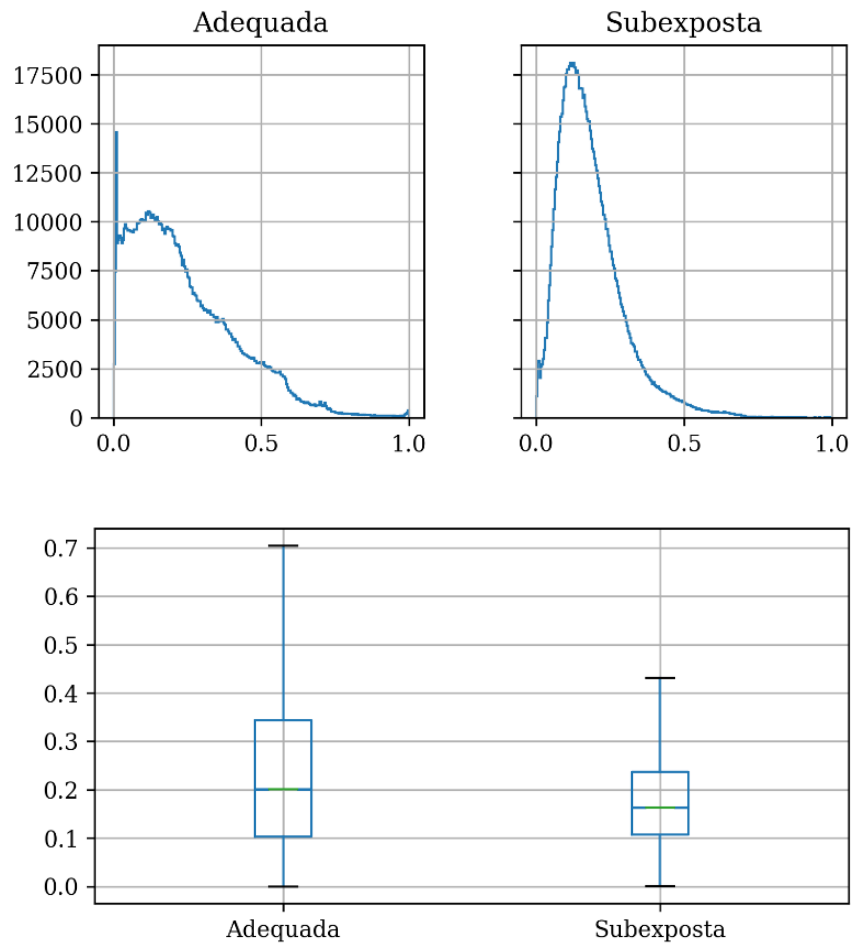

Figura 4: Distribuição dos valores de intensidade de píxel para imagens de cenas do Dataset 2: Fuji para condição adequada e subexposta. 
As Figuras 3 e 4 apresentam ainda os histogramas e diagramas de caixa para os 2 datasets, considerando as imagens com exposição adequada. Cabe ressaltar que a condição considerada adequada não reflete uma condição ideal, mas sim aquela em que se obtém visibilidade razoável da cena sem interferir nas suas condições de iluminação, respeitando os limites dos sensores.

Através desta verificação inicial, pode-se observar que diferentes cenas e parâmetros do equipamento de aquisição resultam em curvas de distribuição significativamente diferentes, havendo pouca similaridade entre os datasets. Pode se observar que a distribuição dos valores de intensidade nas imagens adequadamente expostas é semelhante à apresentada em imagens subexpostas, destacando-se um aumento na média e no desvio padrão. A variação na média e desvio padrão refletem-se visualmente nas imagens como uma melhor distribuição da luminância no intervalo de representação.

A Tabela 1 complementa as informações, mostrando um padrão de tendência central em que a mediana é inferior à média. Observa-se ainda que a maior parte dos valores estão localizados abaixo da metade do intervalo de representação utilizado, com o primeiro quartil e terceiro quartil abaixo do valor de intensidade 0.5. O padrão de assimetria positiva apresentado reflete a distribuição visualizada nos histogramas. Os resultados do teste Jarque-Bera mostram um valor-p muito próximo a zero, levando a rejeição da $\mathrm{H}_{0}$ de que os dados apresentam distribuição normal.

Tabela 1: Distribuição dos valores de píxel para imagens apropriadamente expostas nos 2 datasets.

\begin{tabular}{ccccc}
\hline & \multicolumn{2}{c}{ Dataset 1: Sony } & \multicolumn{2}{c}{ Dataset 2: Fuji } \\
\cline { 2 - 5 } & Adequada & Subexposta & Adequada & Subexposta \\
\hline Média & 0,290 & 0,227 & 0,298 & 0,255 \\
Desvio Padrão & 0,210 & 0,182 & 0,207 & 0,173 \\
Mediana & 0,245 & 0,188 & 0,258 & 0,213 \\
Q1 & 0,117 & 0,076 & 0,135 & 0,132 \\
Q3 & 0,429 & 0,347 & 0,429 & 0,324 \\
Menor & 0,000 & 0,000 & 0,000 & 0,000 \\
Maior & 1,000 & 1,000 & 1,000 & 1,000 \\
Assimetria & 0,793 & 0,777 & 0,753 & 1,303 \\
Curtose & 0,081 & $-0,131$ & $-0,001$ & 1,472 \\
Jarque-Bera (valor-p) & 0,000 & 0,000 & 0,000 & 0,000 \\
\hline
\end{tabular}

A Tabela 2 apresenta os resultados da análise de correlação e dos testes não paramétricos aplicados aos dados, tendo em vista a identificação de evidências de não normalidade. Os coeficientes de correlação de Spearman indicam correlação positiva e moderada-forte entre os pares testados de imagem subexposta e a imagem com exposição adequada em ambos datasets. Os valores-p associados aos coeficientes de correlação de Spearman são inferiores a 0,0001, em ambos datasets, indicando que é improvável que não exista associação entre os dois tipos de imagem.

Nos dois conjuntos de dados, o teste de Wilcoxon indicou um valor-p ínfimo $(<0,0001)$, encontrando evidências para rejeitar a ideia de que a diferença entre os conjuntos ocorre por acaso em ambos datasets, levando a conclusão de que é possível considerar que cada subconjunto é originado de população com medianas distintas. Por fim, para no teste de Levene, obteve-se um valor-p < 0,0001, identificando evidências de que heterocedasticidade entre as variâncias. Evidencia-se que, se fossem desconsideradas as propriedades dos dados que levaram à realização dos testes não paramétricos, os resultados obtidos com os testes paramétricos seriam os mesmos.

A equivalência entre os resultados para o teste $\mathrm{T}$ de Student e para o teste de Wilcoxon também é verificada para este conjunto de dados. Nos três testes realizados, observa-se um valor-p ínfimo, rejeitando a ideia de que a diferença entre os conjuntos ocorre por acaso, levando a conclusão de que cada subconjunto é originado de populações com medianas distintas. 
Tabela 2: Correlação e testes não paramétricos para os subconjuntos de cada dataset.

\begin{tabular}{ccc}
\hline & $\begin{array}{c}\text { Dataset 1: Sony } \\
\text { Subexposta vs Adequada }\end{array}$ & $\begin{array}{c}\text { Dataset 2: Fuji } \\
\text { Subexposta vs. Adequada }\end{array}$ \\
\hline Número de Observações (Píxeis) & 12262410 & 46900737 \\
Corr. Pearson & $0,752(0,00)$ & $0,660(0,00)$ \\
Corr. Spearman & $0,666(0,00)$ & $0,586(0,00)$ \\
Teste $\boldsymbol{t}$ pareado (valor-p) & 0,000 & 0,000 \\
Wilcoxon-Pratt (valor-p) & 0,000 & 0,000 \\
Wilcoxon (valor-p) & 0,000 & 0,000 \\
Ansari-Bradley (valor-p) & 0,997 & 0,985 \\
Levene (valor-p) & 0,000 & 0,000 \\
\hline
\end{tabular}

O teste Ansari e Bradley (1960) [15] para duas amostras é uma alternativa não-paramétrica ao teste $\mathrm{F}$ para variâncias iguais. $\mathrm{O}$ valor-p encontrado para todas as comparações indica que a proporção verdadeira de escalas não é igual a 1. Para o teste de Wilcox on, o menor valor-p indica que a probabilidade de obter um valor como o observado é improvável, levando à rejeição da hipótese nula. Por fim, para o teste de Levene, obteve-se um valor-p 0.00 , menor que o nível de significância $\alpha=0,05$ escolhido, portanto rejeita-se a hipótese de igualdade das variâncias.

\section{CONCLUSÃO}

O presente trabalho apresenta uma avaliação estatística das propriedades de imagens digitais obtidas sob distintas condições de exposição. Destaca-se que, não são conhecidos trabalhos anteriores que compartilhem objetivos ou tenham aplicado estatística inferencial sobre os conjuntos de dados aqui estudados. Utilizando uma abordagem que trabalha a luminância como variável estatística contínua identificou-se, de maneira geral, concordância entre testes paramétricos e nãoparamétricos.

Destaca-se o impacto das condições que compõe o triângulo da exposição na formação da imagem. Para uma mesma cena, distintas condições de exposição podem resultar em diferenças significativas, levando alguns testes a concluir que as amostras são oriundas de populações diferentes. Em condições de baixa iluminação, a relação sinal ruído é mais perceptível, uma vez que a irradiância da cena é baixa para os sensores utilizados. Os resultados encontrados fornecem indicativos de que testes estatísticos simples permitem identificar padrões visuais, corroborando aspectos elencados na bibliografia relacionada.

Em trabalhos futuros, considera-se a aplicação de testes estatísticos e sua implementação como parte integrante de modelos de redes neurais artificiais profundas ou como função objetivo. Como parte integrante do modelo, objetiva-se verificar o impacto de transformações na distribuição dos dados no processamento. Já como função objetivo, pretende-se verificar a viabilidade de suplantar técnicas computacionalmente custosas, como GAN's, por testes estatísticos mais simples, intuitivos e depuráveis. A aplicação de testes estatísticos como função objetivo, se bem-sucedida, pode ainda facilitar o treinamento em dados não pareados, uma vez que permitiria substituir métricas píxel-apíxel (erro médio quadrático, erro absoluto) por testes que verificam a diferença entre a distribuição referência e a distribuição resultante do modelo. Espera-se através de tal implementação reduzir o custo computacional associado ao treinamento, acelerar a convergência do modelo ou até mesmo aumentar a robustez dos modelos treinados.

\section{AGRADECIMENTOS}

O presente trabalho foi realizado com apoio da Coordenação de Aperfeiçoamento de Pessoal de Nível Superior - Brasil (CAPES) - Código de Financiamento 001. 


\section{REFERÊNCIAS BIBLIOGRÁFICAS}

1. Forsyth DA, Ponce J. Computer Vision: A Modern Approach. Pearson Education Limited; 2015.

2. Goodfellow I, Pouget-Abadie J, Mirza M, Xu B, Warde-Farley D, Ozair S. Generative adversarial nets. In: Advances in neural information processing systems; 2014. p. 2672-2680.

3. Simoncelli EP, Olshausen BA. Natural image statistics and neural representation. Annu Rev Neurosci. 2001;24(1):1193-1216, doi: 10.1146/annurev.neuro.24.1.1193.

4. Torralba A, Oliva A. Statistics of natural image categories. Network: computation in neural systems. 2003;14(3):391-412, doi: 10.1088/0954898x_14_3_302.

5. Geisler WS. Visual perception and the statistical properties of natural scenes. Annu Rev Psychol. 2008;59:167-192, doi: 10.1146/annurev.psych.58.110405.085632.

6. Motoyoshi I, Nishida S, Sharan L, Adelson EH. Image statistics and the perception of surface qualities. Nature. 2007;447(7141):206, doi: 10.1038/nature05724.

7. He K, Sun J, Tang X. Single image haze removal using dark channel prior. IEEE Trans Pattern Anal Mach Intell. 2011;33(12):2341-2353, doi: 10.1109/TPAMI.2010.168.

8. Miao L, Chen Y, Wang A. Video smoke detection algorithm using dark channel priori. In: Control Conference (CCC), 2014 33rd Chinese. IEEE; 2014. p. 7405-7408, doi: 10.1109/ChiCC.2014.6896230.

9. Long C, Zhao J, Han S, Xiong L, Yuan Z, Huang J. Transmission: a new feature for computer vision based smoke detection. In: International Conference on Artificial Intelligence and Computational Intelligence. Springer; 2010. p. 389-396, doi: 10.1007/978-3-642-16530-6 46.

10. Wang Z, Bovik AC, Sheikh HR, Simoncelli EP. Image quality assessment: from error visibility to structural similarity. IEEE Trans Image Process. 2004;13(4):600-612, doi: 10.1109/TIP.2003.819861.

11. Wang Z, Simoncelli E, Bovik A. Multi-scale structural similarity for image quality assessment. In: Asilomar Conference on Signals Systems and Computers. vol. 2. IEEE; 1998; 2003. p. 1398-1402, doi: 10.1109/ACSSC.2003.1292216.

12. Chen C, Chen Q, Xu J, Koltun V. Learning to See in the Dark. In: 2018 IEEE/CVF Conference on Computer Vision and Pattern Recognition. IEEE; 2018. p. 3291-3300, doi: 10.1109/cvpr.2018.00347.

13. Jarque CM, Bera AK. Efficient tests for normality, homoscedasticity and serial independence of regression residuals. Econ Lett. 1980;6(3):255-259, doi: 10.1016/0165-1765(80)90024-5.

14. Benesty J, Chen J, Huang Y, Cohen I. Pearson correlation coefficient. In: Noise reduction in speech processing. Springer; 2009. p. 1-4, doi: 10.1007/978-3-642-00296-0 5.

15. Ansari AR, Bradley RA. Rank-sum tests for dispersions. Ann Mathem Statist. 1960 ;31(4):1174-1189, doi: 10.1214/aoms/1177705688.

16. Sprent P, Smeeton NC. Applied nonparametric statistical methods. Chap- man and Hall/CRC; 2000, doi: $10.1201 / 9781420057713$.

17. Olkin I. Contributions to probability and statistics: essays in honor of Harold Hotelling. Stanford University Press; 1960, doi: 10.2307/2004242.

18. Brown MB, Forsythe AB. Robust tests for the equality of variances. J Am Stat Assoc. 1974;69(346):364367, doi: $10.2307 / 2285659$. 